\title{
High energy $\gamma$-ray emission from PSR B1259-63 during 2014 and 2010 periastron passages
}

\author{
Giuseppe Andrea Caliandro* \\ W. W. Hansen Experimental Physics Laboratory, Kavli Institute for Particle Astrophysics and \\ Cosmology, Department of Physics and SLAC National Accelerator Laboratory, Stanford \\ University, US. Consorzio Interuniversitario per la Fisica Spaziale (CIFS), Italy \\ E-mail: caliandrdslac.stanford.edu

\section{C. Teddy Cheung}

Space Science Division, Naval Research Laboratory, USA

Jian Li

Institut de Ciències de l'Espai (IEEC-CSIC), Spain

\section{Diego F. Torres}

Institut de Ciències de l'Espai (IEEC-CSIC), Spain Inst. Catalana de Recerca i Estudis Avancats, Spain

\section{Kent Wood}

Space Science Division, Naval Research Laboratory, USA

PSR B1259-63/LS 2883 is a $\gamma$-ray binary system composed of a radio pulsar in a long (1236.7 days) and elliptical ( $e \sim 0.87$ ) orbit around a Be star. In its 2010 periastron passage, multiwavelength emission from radio to $\mathrm{TeV}$ was observed, and an unexpected $\mathrm{GeV}$ flare was detected by the Fermi Large Area Telescope (LAT). Here we present the results of the LAT monitoring of PSR B1259-63 during its most recent 2014 periastron passage. We confirm that the GeV flare is recurrent within the orbit. The comparison of the 2014 and 2010 periastron passages shows overall similarities of flare durations, average flux levels, and spectra. In contrast, the detailed time evolutions of the two flares present interesting differences. Indeed, the light curves of the two flares show both a different structure and peak energy flux $\left(9.6 \pm 1.8 \times 10^{-10} \mathrm{erg} \mathrm{cm}^{-2} \mathrm{~s}^{-1}\right.$ and $7.1 \pm 1.3 \times 10^{-10} \mathrm{erg} \mathrm{cm}^{-2} \mathrm{~s}^{-1}$, respectively in 2010 and 2014). While the tail of the 2010 flare the flux decayed exponentially, in 2014 it persisted at a high level. The interpretation of these differences as well as of the flare themselves is subject of debate.

Swift: 10 Years of Discovery,

2-5 December 2014

La Sapienza University, Rome, Italy

\footnotetext{
* Speaker.
} 


\section{Introduction}

PSR B1259-63/LS 2883 is one of the few binaries systems belonging to the class of the " $\gamma$-ray binaries". This class is a subsample of the High Mass X-ray Binaries (HMXB) with the peculiar characteristic that the $\gamma$-ray emission dominates the radiative spectral energy distributions

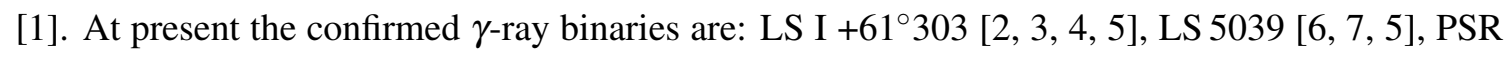

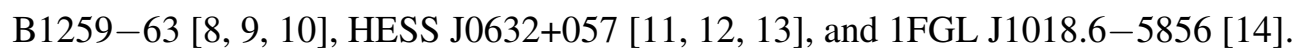

Among them, PSR B1259-63 is the only one whose compact object is known to be a radio pulsar. It is a young pulsar with spinning period $P=47.76 \mathrm{~ms}$ [[15], that moves in a very eccentric orbit ( $e \sim 8.7$ ) around the massive star LS 2883, with orbital period of 1236.7 days ( 2.4 years) [ए6]. LS 2883 is a massive Be star $\left(M \sim 30 M_{\odot}\right)$ with an equatorial disk inclined with respect to the orbital plane, such that the pulsar crosses the disk just before and just after each periastron passage [ए]].

The powerful pulsar wind generated by the pulsar interacts with the stellar disk during the passages through it, giving rise to a broadband non-thermal unpulsed emission in the radio (e.g.

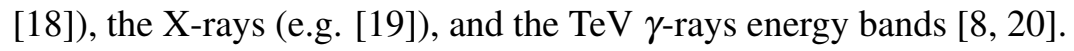

In the $\mathrm{GeV}$ energy band, the first periastron passage observed by Fermi-LAT occurred in December 2010 [Q, [0]]. The behavior of the source in this energy range was surprisingly different from the other wavelengths. Before and during the periastron passage, the LAT detected just a weak emission above $100 \mathrm{MeV}$, while $\sim 30$ days after the periastron a very bright flare was detected, with a flux about 10 times the pre-periastron value. The flares continued until about three months after the periastron passage.

The radiation mechanisms of the broadband emission from PSR B1259-63/LS 2883, as well as the GeV flare is still under debate (e.g. [2], [22, 221, 24] ).

After its 3.4-year orbit period, PSR B1259-63 approached periastron again on 2014 May

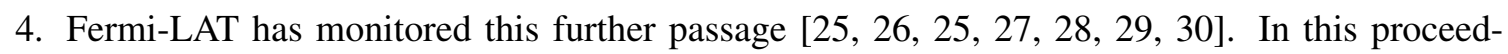
ing, Fermi-LAT analysis results of PSR B1259-63 over the last periastron passage are presented, together with the comparison and a new analysis of the periastron passage occurred from 2010.

\section{Periastron passages during Fermi-LAT activity}

Since the launch of the Fermi satellite PSR B1259-63 undergo two periastron passages, on 2010, and more recently on 2014. Precisely, as derived from the most recent measurement of orbital parameters [ए6], the 2010 and 2014 periastron time are MJD 55544.693781 (2010 December 14 16:39:02.678 UT) and MJD 56781.418307 (2014 May 4 10:02:21.725 UT). We analyzed FermiLAT data since 2 months prior to each periastron up to three months after. The analysis is performed selecting from the Pass 7 reprocessed data SOURCE class events which fall in a circular region of $10^{\circ}$ radius centered on PSR B1259-63, and within an energy range of 0.1-100 GeV. To reject contaminating gamma rays from the Earth's limb, we selected events with zenith angle smaller than $100^{\circ}$. The light curves presented here are calculated performing a binned maximum likelihood fit in each time bin using the Science Tool gtlike. The spectral-spatial model constructed to perform the likelihood analysis includes Galactic and isotropic diffuse emission components as well as known $\gamma$-ray sources within $15^{\circ}$ of PSR B1259-63 based on the third Fermi-LAT source catalog [B]]. 

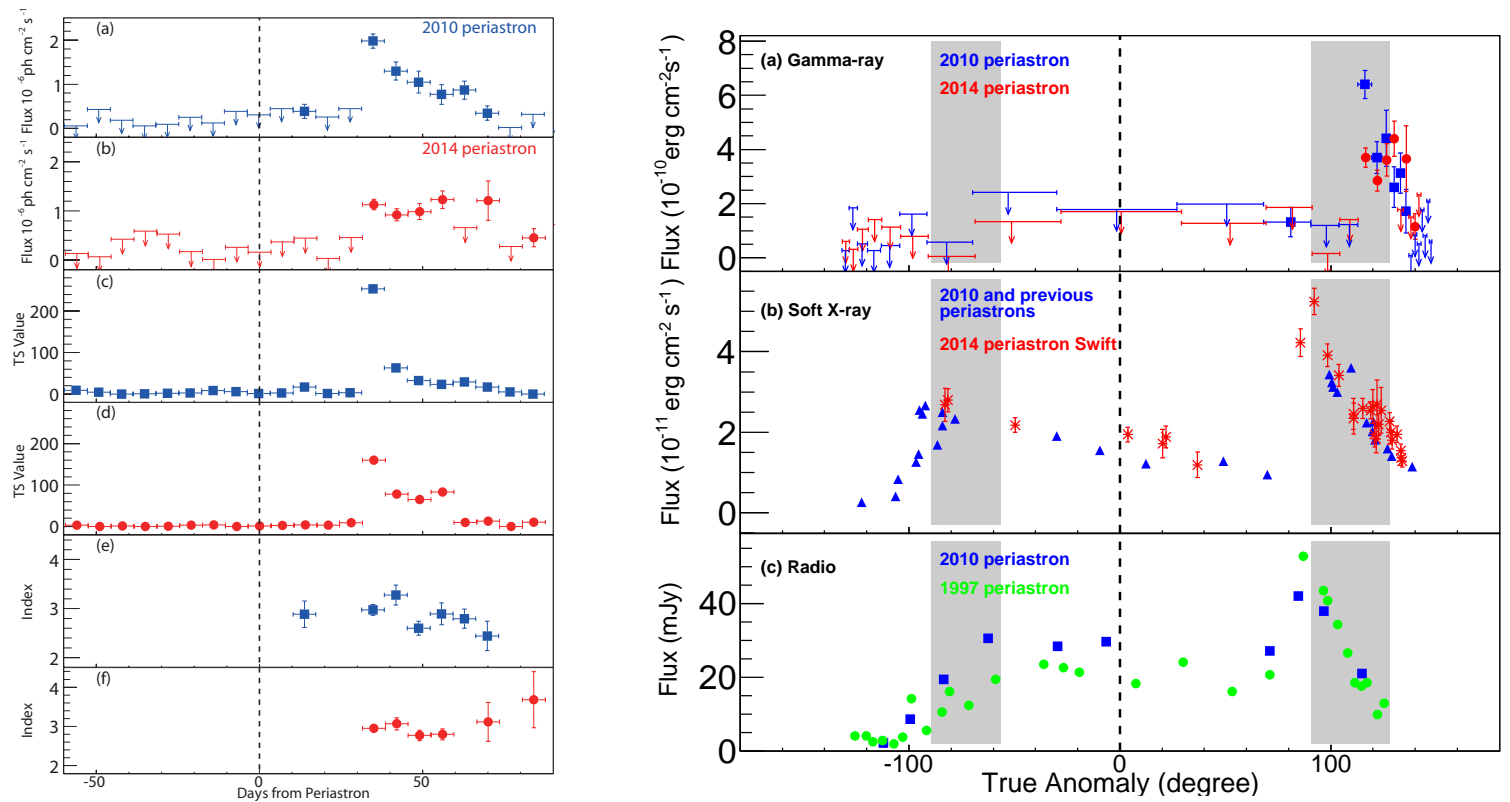

Figure 1: Preliminary. Left. Weekly $\gamma$-ray flux for $E>100 \mathrm{MeV}$ (a \& b), TS value (c \& d), and photon index (e \& f) of PSR B1259-63 during the 2010 (blue) and 2014 (red) periastron passages. The dashed black line shows the time of periastron. Right. (a) Weekly $\gamma$-ray flux of 2010 (blue) and 2014 (red) periastron passage in energy units. (b) X-ray fluxes from 2014 Swift (red) and previous periastron passages (blue, 2004, 2007 and 2010 periastra from [ㅁ]). (c) Radio (2.4 GHz) flux densities for the 2010 (blue) and 1997 periastron passages (green, [Q] ). X axis is in true anomaly. The dashed black line shows the time of periastron. Shaded area corresponds to the Be circumstellar disk position proposed in [34].

The spectral parameters were fixed to the catalog values, except for the sources within $3^{\circ}$ of PSR B1259-63. For these latter sources, the flux normalization was left free. PSR B1259-63 itself was modeled as a single power-law with all spectral parameters allowed to vary. For the time bins with low detection significance (Test Statistic value TS $<9$ ), we placed a 95\% upper limit on the PSR B1259-63 photon flux above $100 \mathrm{MeV}$, as evaluated with Helene's method [B2].

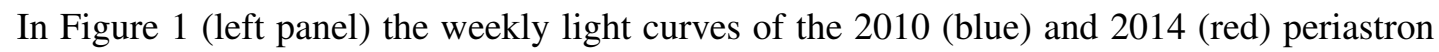
passages are swhown. During both passages, a bright flaring activity below $1 \mathrm{GeV}$ was detected starting $\sim 30$ days after the periastron.

It is important to highlight that on 2014 Fermi-LAT performed a Target of Opportunity (ToO) observation of PSR B1259-63 starting 27 days after the periastron passage, and lasting 19 days. Thus, the prompt of the gamma-ray activity was observed with an exposure of PSR B1259-63 increased by a factor of $\sim 2$ compared to normal observations.

The analysis performed here shows consistent results with those reported in [Q], in which the analysis was carried out with Pass 6 data.

\section{Similarities and differences between 2014 and 2010 flares}

The onset of both 2010 and 2014 flares is after $\sim 30$ days from the periastron. They have a similar duration of $\sim 40 \mathrm{~d}$. The flaring is characterized by a sudden high flux increase followed by a tail, which on 2010 decrease exponentially, while in 2014 is flatter, as shown in Figure $\mathbb{W}$ left panel. 
In order to study similarities and differences between the 2014 and 2010 flare profiles, their smoothed light curves are plotted in Figure $\square$. The smoothed light curves were produced using a sliding window technique. We chose a time window of 3 days moving forward in time with steps of 3 hours. For each step, a binned likelihood analysis was performed on the time window. The spectral index of PSR B1259-63 was allowed to vary between the values 2.0 and 3.5. The shaded areas in Figure $\square$ around the solid lines correspond to the statistical error of the flux calculation. When the TS value is low (TS $<9$ ), the smoothed light curve points are represented as null flux with shaded areas showing the upper limits of $95 \%$ confidence level.

After the onset, the 2010 periastron smoothed flux steeply increases up to its maximum at $\sim 36$ days. However, in 2014 the increase is delayed by forming a short plateau until $\sim 34$ days, after which the flux rises up to the peak (at $\sim 38$ days).

The peak flux of 2014 periastron is lower than the 2010 periastron. Indeed, the 2014 periastron highest day-average flux was $7.1 \pm 1.3 \times 10^{-10} \mathrm{erg} \mathrm{cm}^{-2} \mathrm{~s}^{-1}$, corresponding to $\sim 50 \%$ of the pulsar spin-down luminosity, $8.3 \times 10^{35} \mathrm{erg} \mathrm{s}^{-1}$ [प[5]. In contrast, the highest day-average flux of 2010 periastron was $9.6 \pm 1.8 \times 10^{-10} \mathrm{erg} \mathrm{cm}^{-2} \mathrm{~s}^{-1}$, corresponding to $\sim 70 \%$ of the pulsar spin-down luminosity.

The main peak of the 2014 profile is delayed by $\sim 2$ days with respect to the 2010 flare. This delay is consistent with the result of the cross-correlation we calculated for the daily light curves of the 2010 and 2014 flaring activity (the daily light curves are shown in [26]).

In 2010 the main peak is followed by a marked second peak (at $\sim 43$ days), which in the 2014 light curve is substituted by a smooth valley.

Finally, the 2014 flaring activity ends with a possible peak at $\sim 68$ days, which is consistent with the last high flux bin of the weekly light curve (see Figure $\mathbb{U}$ ). However, the TS value of this bin is very low $(\mathrm{TS}<20$ ). We have also noticed that the exposure of PSR B1259-63 reaches its minimum exactly in correspondence of this final peak. It is $\sim 5$ times less than the exposure of the main peak. Therefore, this final peak can be just an artifact due to the very low exposure.

\section{Multi-Wavelength context}

During its 2014 periastron PSR B1259-63 was monitored also by Swift/XRT in Photon Counting (PC) and Window Timing (WT) modes. We analyzed the Swift/XRT 0.1-10 keV data. A detailed description of the analysis is given in [[26].

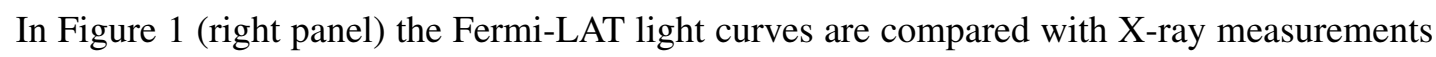
of PSR B1259-63 during 2014 and previous periastra, and radio measurements during 2010 and 1997 periastra.

The Radio and X-ray flux profiles show an enhancement either before and after the periastron, which is expected when the pulsar crosses the companion's circumstellar disk twice each orbit

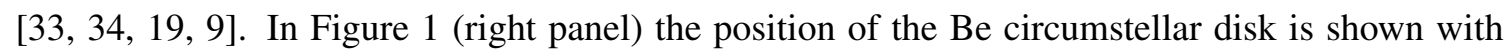
shaded areas as proposed in [B4]].

In contrast, the $\gamma$-ray flaring activity was detected only after the periastron. Although the 2010 and 2014 flares are within the second crossings of the Be circumstellar disk, they are not in line with X-ray and radio activities. Indeed, the prompt of the $\gamma$-ray flares are delayed respect to the other wavelengths by $\sim 30$ true anomaly degree, corresponding to $\sim 15$ days. 


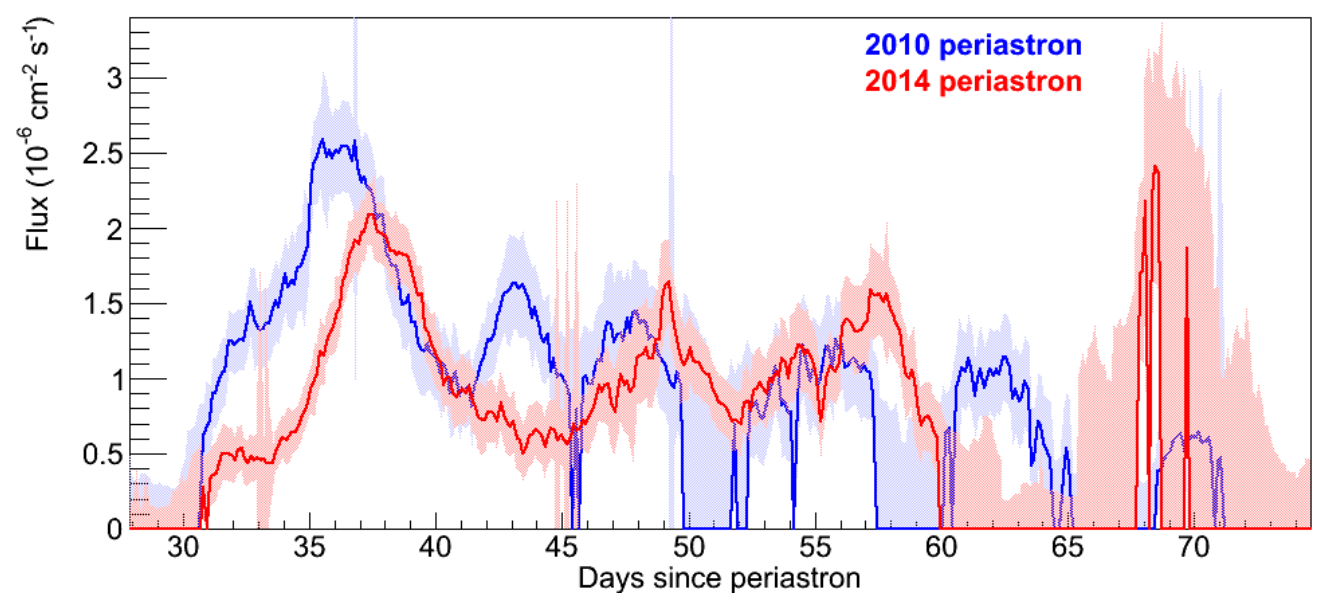

Figure 2: Preliminary. Flux smoothed light curves of the 2010 and 2014 flaring activities after periastron (blue and red, respectively) produced with a sliding window. The shaded areas demonstrate the statistical error of the flux. The smoothed light curve points are represented as null flux when the TS value is below 9, with only shaded areas showing the upper limits of $95 \%$ confidence.

\section{Summary}

The observations of 2010 and 2014 periastron passages of PSR B1259-63 with Fermi-LAT has demonstrated the recurrent flaring behavior of the gamma-ray emission after the periastron passage. We have shown that the two passages manifest some striking similarities. At the same time, there are also certain differences which may be due to inhomogeneities in the shape, density, or extent in the circumstellar disk of the Be star. The two flares were characterized by a high efficiency of conversion from pulsar spin-down power into gamma-ray emissions. Though we have now observed two similar GeV flares from PSR B1259-63, the origin of these events is still unclear. To explain it authors have considered up scattering of the circumstellar disk IR photons by the unshocked pulsar wind electrons [ [2]], or Doppler boosted synchrotron emission in the bowshock tail [D2], or also up-scattering of X-ray photons from the surrounding pulsar wind nebula [23] .

\section{Acknowledgements}

The Fermi-LAT Collaboration acknowledges support for LAT development, operation and data analysis from NASA and DOE (United States), CEA/Irfu and IN2P3/CNRS (France), ASI and INFN (Italy), MEXT, KEK, and JAXA (Japan), and the K.A. Wallenberg Foundation, the Swedish Research Council and the National Space Board (Sweden). Science analysis support in the operations phase from INAF (Italy) and CNES (France) is also gratefully acknowledged.

\section{References}

[1] G. Dubus, Gamma-ray binaries and related systems, $A \& A R v, 21,(2013), 64$ 
[2] J. Albert, et al. Variable Very-High-Energy Gamma-Ray Emission from the Microquasar LS I +61 303, Science, 312, (2006), 1771

[3] V. A. Acciari, et al. VERITAS Observations of the $\gamma$-Ray Binary LS I +61 303, ApJ, 679, (2008), 1427

[4] A. A. Abdo, et al. Fermi LAT Observations of LS I +61 ${ }^{\mathrm{deg}} 303$ : First Detection of an Orbital Modulation in GeV Gamma Rays, ApJL, 701, (2009), L123

[5] D. Hadasch, et al. Long-term Monitoring of the High-energy $\gamma$-Ray Emission from LS I +61 ${ }^{\mathrm{deg}} 303$ and LS 5039, ApJ, 749, (2012), 54

[6] F. Aharonian, et al. Discovery of Very High Energy Gamma Rays Associated with an X-ray Binary, Science, 309, (2005), 746

[7] A. A. Abdo, et al. Fermi/LAT observations of LS 5039, ApJL, 706, (2009), L56

[8] F. Aharonian, et al. Discovery of the binary pulsar PSR B1259-63 in very-high-energy gamma rays around periastron with HESS, A\&A, 442, (2005), 1

[9] A. A. Abdo, et al. Discovery of High-energy Gamma-ray Emission from the Binary System PSR B1259-63/LS 2883 around Periastron with Fermi, ApJL, 736, (2011), L11

[10] P. H. T. Tam, et al. Discovery of GeV $\gamma$-ray Emission from PSR B1259-63/LS 2883, ApJL, 736, (2011), L10

[11] F. A. Aharonian, et al. Discovery of a point-like very-high-energy $\gamma$-ray source in Monoceros, A\&A, 469, (2007), L1

[12] J. A. Hinton, et al. HESS J0632+057: A New Gamma-Ray Binary?, ApJL, 690, (2009), L101

[13] S. D. Bongiorno, et al. A New TeV Binary: The Discovery of an Orbital Period in HESS J0632+057, ApJL, 737, (2011), L11

[14] Fermi LAT Collaboration, et al. Periodic Emission from the Gamma-Ray Binary 1FGL J1018.6-5856, Science, 335, (2012), 189

[15] S. Johnston, R. N. Manchester, A. G. Lyne, L. Nicastro, \& J. Spyromilio Radio and Optical Observations of the PSR:B1259-63 / SS:2883 Be-Star Binary System, MNRAS, 268, (1994), 430

[16] R. M. Shannon, S. Johnston, \& R. N. Manchester The kinematics and orbital dynamics of the PSR B1259-63/LS 2883 system from 23 yr of pulsar timing, MNRAS, 437, (2014), 3255

[17] N. Wex, et al. Timing models for the long orbital period binary pulsar PSR B1259-63, MNRAS, 298, (1998), 997

[18] S. Johnston, L. Ball, N. Wang, \& R. N. Manchester Radio observations of PSR B1259-63 through the 2004 periastron passage, MNRAS, 358, (2005), 1069

[19] M. Chernyakova, A. Neronov, F. Aharonian, Y. Uchiyama, \& T. Takahashi X-ray observations of PSR B1259-63 near the 2007 periastron passage, MNRAS, 397, (2009), 2123

[20] F. Aharonian, et al. Very high energy $\gamma$-ray observations of the binary PSR B1259-63/SS2883 around the 2007 Periastron, A\&A, 507, (2009), 389

[21] D. Khangulyan, F. A. Aharonian, S. V. Bogovalov, \& M. Ribó; Post-periastron Gamma-Ray Flare from PSR B1259-63/LS 2883 as a Result of Comptonization of the Cold Pulsar Wind, ApJL, 752, (2012), L17 
[22] S. W. Kong, K. S. Cheng, \& Y. F. Huang Modeling the Multiwavelength Light Curves of PSR B1259-63/LS 2883. II. The Effects of Anisotropic Pulsar Wind and Doppler Boosting, ApJ, 753, (2012), 127

[23] G. Dubus \& B. Cerutti What caused the GeV flare of PSR B1259-63?, A\&A, 557, (2013), A127

[24] M. Chernyakova, et al. Multiwavelength observations of the binary system PSR B1259-63/LS 2883 around the 2010-2011 periastron passage, MNRAS, 439, (2014), 432

[25] P. H. T. Tam, et al. High-energy Observations of PSR B1259-63/LS 2883 through the 2014 Periastron Passage: Connecting X-Rays to the GeV Flare, ApJL, 798, (2015), L26

[26] G. A. Caliandro et al. Gamma-ray flare activity from PSR B1259-63 during 2014 periastron passage and comparison to its 2010 passage, ApJ, accepted for publication, (2015)

[27] D. Malyshev, A. Neronov, \& M. Chernyakova Flaring activity from PSR B1259-63, The Astronomer's Telegram, 6204, (2014), 1

[28] P. H. T. Tam, A. K. H. Kong, \& G. C. K. Leung Fermi LAT detection of a flaring activity from PSR B1259-63/LS 2883, The Astronomer's Telegram, 6216, (2014), 1

[29] K. S. Wood, et al. Onset and Rapid Increase of Gamma-Ray activity from the Binary System PSR B1259-63 detected by Fermi LAT, The Astronomer's Telegram, 6225, (2014), 1

[30] C. Pittori, et al. AGILE detection of a gamma-ray flare from the PSR B1259-63 region, The Astronomer's Telegram, 6231, (2014), 1

[31] The Fermi-LAT Collaboration Fermi Large Area Telescope Third Source Catalog, ApJS, submitted, (2015), [arXiv:1501.02003]

[32] O. Helene, Upper limit of peak area, Nuclear Instruments and Methods in Physics Research, 212, (1983), 319

[33] L. Cominsky, M. Roberts, \& S. Johnston Detection of X-ray emission from the PSR 1259-63/SS 2883 binary system, ApJ, 427, (1994), 978

[34] M. Chernyakova, A. Neronov, A. Lutovinov, J. Rodriguez, \& S. Johnston XMM-Newton observations of PSR B1259-63 near the 2004 periastron passage, MNRAS, 367, (2006), 1201 